\title{
OPTIMIZATION OF THE SWINGING MODE OF THE BOOM CRANE UPON A COMPLEX INTEGRAL CRITERION
}

\author{
VIATCHESLAV LOVEIKIN, YURIY ROMASEVYCH*, \\ IVAN KADYKALO, ANASTASIA LIASHKO \\ Department of Machines and Equipment Design, National University of Life \\ and Environmental Sciences of Ukraine, Kyiv, 03041, Ukraine
}

[Received: 16 April 2019. Accepted 08 July 2019]

doi: 10.7546/JTAM.49.19.03.07

\begin{abstract}
The article presents the method of solving the problem of elimination of load oscillations on a flexible suspension during the swing of a boom crane with simultaneous minimization of dynamic forces in the transmission mechanism. A complex integral dynamic criterion to minimize includes the root-mean-square value of an elastic torque in the drive mechanism and the rate of its changing. The solution of the optimization problem is represented in the discrete form. The multi-epoch particle swarm optimization (ME-PSO) method has been used for that purpose. It helped to obtain the discrete values of the kinematic, force and power characteristics of the boom system. The resultant optimal mode of the boom-system swing has improved crane performance in terms of reliability and energy efficiency.
\end{abstract}

KEY WORDS: boom crane, criterion, dynamic forces, load, minimization, swing mechanism, slew drive, oscillations, optimization.

\section{INTRODUCTION}

\subsection{PRoblem STATEMENT}

The operation of the boom crane swing mechanism results in load oscillations on a flexible suspension and large dynamic forces on the elements of the drive mechanism and the metal structures of the crane [1-4]. These loads are especially dangerous during the transient processes of the swing mechanism (starting and braking). It is proposed that in order to eliminate the load oscillations and to reduce the dynamical forces, the starting process should be optimized [5-7].

It is reasonable to use a complex integral criterion, which takes into account the effect of the dynamic forces in the crane swing drive mechanism and their rate. The latter has a significant influence on the occurrence of oscillations in the drive mechanism and the load on a flexible suspension. While solving the optimization problem, the problem of minimizing the complex nonlinear integral criterion (functional) arises

\footnotetext{
${ }^{*}$ Corresponding author e-mail: romasevichyuriy@ukr.net
} 
that cannot be solved using the existing analytical and numerical methods. One of the ways to solve the above problem is to use a metaheuristic algorithm [8], in particular, the ME-PSO method $[9,10]$ or other similar methods. The solution of this problem will make it possible to apply the optimization methods for the motion modes of nonlinear mechanical systems.

\subsection{ANALYSIS OF RECENT STUDIES AND PUBLICATIONS}

The expansion of the scope of activities and the emergence of new lines of research and new, more difficult problems contribute to the need for other methods to solve problems of increased complexity and dimensionality. This requires further improvement of combinatorial optimization methods and the development of new mathematical optimization models, which may be applied in present-day information technologies [8].

Studies [8-10] provide the findings of recent research on the development and implementation of applied combinatorial optimization methods, the issues of formalization, classification, and assessment of the computing complexity of combinatorial optimization problems, and state-of-the-art approaches to the solution of the above problems. The main focus is on metaheuristic methods. It has been confirmed that combinatorial optimization methods can be used to solve a wide range of applied problems arising in science, technology, biology, economics, production, etc. Optimization by means of natural (biological) methods is becoming widely spread in the various domains of human activities [9].

One of the methods applied for the solution of this problem is the particle swarm optimization (PSO) method, which simulates a school of fish or a flock of birds [10].

The authors of [11] have analysed the genetic algorithm, the PSO algorithm and the neuron-genetic method for the solution of the problem.

The PSO method is used to calculate various control problems, develop artificial neural networks, process signals, etc. [10,12-15].

Authors in [16] propose a new technology based on the particle swarm optimization technology. The basic idea consists in the re-initiation of the stagnation swarm with low intelligence efficiency.

Apparently, the use of the swarm-based technologies, the PSO method or other similar methods and their modifications [8-17] makes it possible to use the optimization methods of the motion modes of nonlinear mechanical systems.

The Purpose Of This Study is to optimize the swinging mode of the boom crane upon a complex integral criterion. To achieve this purpose, the following problems need to be solved: 1) to choose the model of the boom crane swing dynamics; 2) to justify the optimization criterion of the crane swing mode and to determine its extreme values; 3 ) to determine the optimal mode of the swing mechanism motion; 
4) to analyze the obtained results.

\section{MAIN TEXT}

While developing the dynamic model of the boom crane swing mechanism, we suppose that the basic elements of the crane are absolutely solid objects, except for the drive mechanism, whose elements have elastic properties, and the load on a flexible suspension, which is represented in the form of a moveable mathematical pendulum. Besides, we ignore the rope's radial vertical deviations that do not depend on the swing mode and are determined by the centrifugal force, so we only take into account the rope's deviation in the direction that is tangential to the circular load motion. Thus, the dynamic model of the boom crane swing mechanism may be represented as a holonomic mechanical system with three degrees of freedom, as shown in Fig. 1 [2].

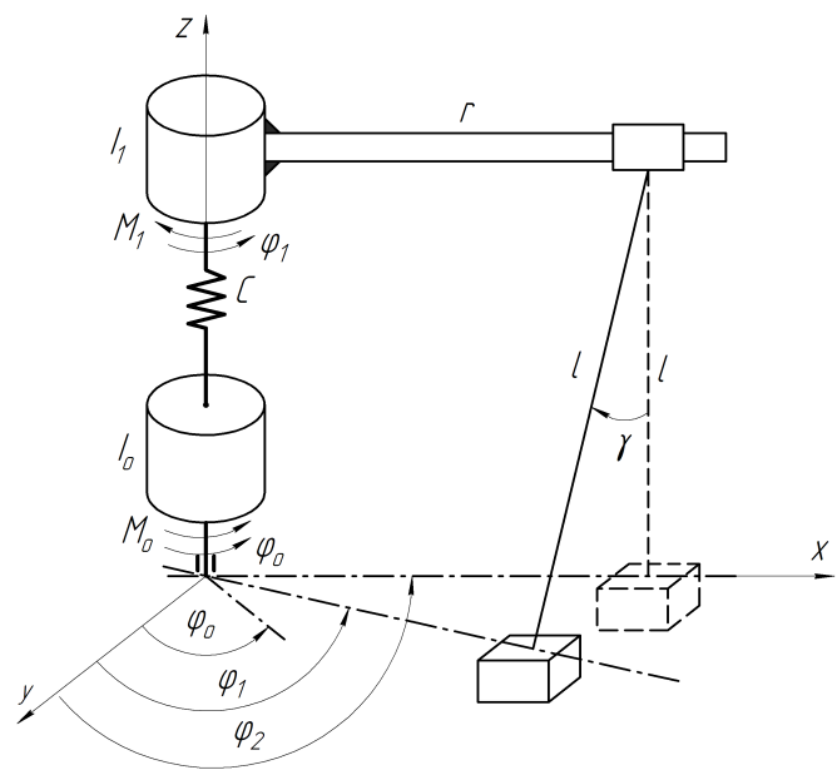

Fig. 1. Dynamic model of the swing mechanism.

The angular coordinates of the rotation angle of the electric motor rotor $\varphi_{0}$, the revolving part of the boom crane $\varphi_{1}$ and the load $\varphi_{2}$ reduced to the axis of rotation of the crane are taken as generalized coordinates.

The presented dynamic model of the crane swing mechanism is definitely matched with the mathematical model in the form of a system of three differential second- 
order equations. They have been obtained with using Lagrange's equations [2]:

$$
\left\{\begin{array}{l}
I_{0} \ddot{\varphi}_{0}=M_{0}-C\left(\varphi_{0}-\varphi_{1}\right) ; \\
I_{1} \ddot{\varphi}_{1}=C\left(\varphi_{0}-\varphi_{1}\right)-m r^{2} \frac{g}{l}\left(\varphi_{1}-\varphi_{2}\right)-M_{1} \\
\ddot{\varphi}_{2}=\frac{g}{l}\left(\varphi_{1}-\varphi_{2}\right),
\end{array}\right.
$$

where $m$ is the mass of the load on a flexible suspension, $I_{0}$ - the inertia moment of the drive mechanism reduced to the revolution axis of the crane, $I_{1}$ - the inertia moment of the revolving part of the crane (boom and tower) relative to its own revolution axis, $M_{0}$ - the breakaway torque on the electric motor shaft reduced to the revolution axis of the crane, $M_{1}$ - the resistance torque in the revolving part of the crane relative to its own revolution axis, $C$ - the rigidity factor of the drive mechanism all elements, reduced to the revolution axis of the crane, $r$ - the length of the boom from the revolution axis of the crane to the point of load suspension on a flexible suspension, $l-$ the length of the flexible load suspension, and $g$ is the free-fall acceleration. Drive mechanism consists of electric motor, coupling, reducer and open gear transmission. The first equation describes the movement of the drive mechanism, the second is related to the rotary part of the crane, and the third equation describes the movement of the load.

Therefore, a complex dimensionless integral dynamic criterion, which takes into account the relative root-mean-square value of elastic torque in the drive mechanism, and its rate have been chosen as the optimization criterion and is represented by the following dependence:

$$
K=\left\{\frac{1}{t_{1}} \int_{0}^{t_{1}}\left[\delta\left(\frac{M_{01}}{M_{n}}\right)^{2}+(1-\delta)\left(\frac{\dot{M}_{01}}{M_{n}} t_{1}\right)^{2}\right] d t\right\}^{1 / 2},
$$

where $t$ is the time, $t_{1}$ - the duration of a transient process (starting, braking), $M_{01}$, $\dot{M}_{01}$ are the elastic torque in the drive mechanism and its rate, respectively, reduced to the crane revolution axis, $M_{n}$ is the torque rating on the drive motor shaft reduced to the crane revolution axis, and $\delta$ is the dimensionless weighting factor that takes into account the proportion of the elastic torque and may vary from 0 to 1 .

Loads emerging during the starting process in the transmission mechanism are significant for the crane swing mechanism, which are represented by the first summand in the integrand of the optimization criterion. In addition, the nature of the change in these loads has a considerable influence on the oscillatory processes that take place in the transmission mechanism and the flexible load suspension, which are 
represented by the second summand of the criterion (2). The importance of the first component is determined by the dimensionless coefficient $\delta$.

Let us determine the constituents of the criterion (2). First, the dependence of the elastic torque in the drive mechanism is found from the second equation of the system (1)

$$
M_{01}=C\left(\varphi_{0}-\varphi_{1}\right)=I_{1} \ddot{\varphi}_{1}+m r^{2} \ddot{\varphi}_{2}+M_{1}
$$

Dependence (3) is reduced only to the generalized coordinate $\varphi_{2}$ and its time derivatives. For this purpose, let us express the coordinate $\varphi_{1}$ from the last equation (1) through coordinate $\varphi_{2}$ and its derivatives; as a result, we obtain

$$
\varphi_{1}=\varphi_{2}+\frac{l}{g} \ddot{\varphi}_{2} .
$$

Having taken time derivatives from expression (4), we shall have

$$
\begin{aligned}
& \dot{\varphi}_{1}=\dot{\varphi}_{2}+\frac{l}{g} \dddot{\varphi}_{2} ; \\
& \ddot{\varphi}_{1}=\ddot{\varphi}_{2}+\frac{l}{g} \stackrel{(4)}{\varphi}_{2} .
\end{aligned}
$$

Having put expression (6) into dependence (3), we shall find

$$
M_{01}=I_{1} \frac{l}{g} \stackrel{(4)}{\varphi}_{2}+\left(I_{1}+m r^{2}\right) \ddot{\varphi}_{2}+M_{1} .
$$

Having taken a time derivative from expression (7), we shall find the rate of the elastic torque change in the drive mechanism

$$
\dot{M}_{01}=I_{1} \frac{l(5)}{g} \stackrel{\varphi}{\varphi}_{2}+\left(I_{1}+m r^{2}\right) \dddot{\varphi}_{2} .
$$

Having put expressions (7) and (8) into criterion (2) and made some transformations, we shall have

(9) $K=\left\{\frac{1}{M_{n}^{2} t_{1}} \int_{0}^{t_{1}}\left\{\delta\left[I_{1} \frac{l}{g} \stackrel{(4)}{\varphi}_{2}+\left(I_{1}+m r^{2}\right) \ddot{\varphi}_{2}\right]^{2}\right.\right.$

$$
\left.\left.+(1-\delta) t_{1}^{2}\left[I_{1} \frac{l\left({ }_{g}\right)}{g}{ }_{2}+\left(I_{1}+m r^{2}\right) \dddot{\varphi}_{2}\right]^{2}\right\} d t\right\}^{1 / 2} .
$$


Let us determine the extreme conditions of the starting process for the dynamic model of the boom system, as shown in Fig. 1:

$$
\left\{\begin{array}{l}
t=0: \quad \varphi_{0}=\varphi_{1}=\varphi_{2}=0, \quad \dot{\varphi}_{0}=\dot{\varphi}_{1}=\dot{\varphi}_{2}=0 \\
t=t_{1}: \quad \varphi_{0}=\varphi_{1}=\varphi_{2}=\omega t_{1} / 2, \quad \dot{\varphi}_{0}=\dot{\varphi}_{1}=\dot{\varphi}_{2}=\omega
\end{array}\right.
$$

where $\omega$ is the set angle rate of the crane swing.

Let us reduce the system of extreme conditions (10) to coordinate $\varphi_{2}$ and its time derivatives. For this purpose, let us express the motor rotor angle coordinate reduced to the crane revolution axis from the second equation of the system (1)

$$
\varphi_{0}=\varphi_{1}+\frac{I_{1}}{C} \ddot{\varphi}_{1}+\frac{m r^{2}}{C} \ddot{\varphi}_{2} .
$$

Using dependencies (4) and (6), expression (11) takes the following form:

$$
\varphi_{0}=\varphi_{2}+\left(\frac{l}{g}+\frac{I_{1}+m r^{2}}{C}\right) \ddot{\varphi}_{2}+\frac{I_{1}}{C} \frac{l}{g} \stackrel{(4)}{\varphi}_{2}+\frac{M_{1}}{C} .
$$

Having taken time derivatives from expression (12), we shall have

$$
\begin{aligned}
& \dot{\varphi}_{0}=\dot{\varphi}_{2}+\left(\frac{l}{g}+\frac{I_{1}+m r^{2}}{C}\right) \dddot{\varphi}_{2}+\frac{I_{1}}{C} \frac{l}{g} \stackrel{(5)}{\varphi}_{2} ; \\
& \ddot{\varphi}_{0}=\ddot{\varphi}_{2}+\left(\frac{l}{g}+\frac{I_{1}+m r^{2}}{C}\right) \stackrel{(4)}{\varphi}_{2}+\frac{I_{1}}{C} \frac{l}{g} \stackrel{(4)}{\varphi}_{2} .
\end{aligned}
$$

Having put expressions (4), (5), (12) and (13) into conditions (10), we have the extreme conditions of the boom system starting process expressed through the coordinate $\varphi_{2}$ and its time derivatives

$$
\left\{\begin{array}{l}
t=0: \quad\left\{\begin{array}{l}
\varphi_{2}=0, \dot{\varphi}_{2}=0, \ddot{\varphi}_{2}=0, \\
\dddot{\varphi}_{2}=0, \stackrel{(4)}{\varphi}{ }_{2}=-\frac{M_{1} g}{I_{1} l}, \stackrel{(5)}{\varphi_{2}}=0 ;
\end{array}\right. \\
t=t_{1}:\left\{\begin{array}{l}
\varphi_{2}=\frac{\omega t_{1}}{2}, \dot{\varphi}_{2}=\omega, \ddot{\varphi}_{2}=0, \\
\dddot{\varphi}_{2}=0, \stackrel{(4)}{\varphi_{2}}=-\frac{M_{1} g}{I_{1} l}, \stackrel{(5)}{\varphi_{2}}=0 .
\end{array}\right.
\end{array}\right.
$$

The condition for the minimum of criterion (9) under extreme conditions (15) is Poisson's equations that cannot be solved analytically. Therefore, in order to solve such a marginal problem, we shall use the approximation methods of swarm-based 
technologies, in particular, the ME-PSO method. For this purpose, let us break the motion interval of the boom system $\left[0, t_{1}\right]$ into discrete points with an interval $\Delta t=$ $\left(t_{n}-t_{0}\right) / n$, where $t_{0}$ and $t_{n}$ are the initial and end points of the motion interval breakdown, and $n$ is the number of intervals.

Let us replace $\varphi_{2}=\varphi, \dot{\varphi}_{2}=\dot{\varphi}, \ddot{\varphi}_{2}=\ddot{\varphi}, \dddot{\varphi}_{2}=\varphi, \stackrel{(4)}{\varphi}{ }_{2}=\stackrel{(4)}{\varphi}, \stackrel{(5)}{\varphi}{ }_{2}=\stackrel{(5)}{\varphi}$ independences (1), ., (15) and replace the continuity of these functions with the approximate discrete values, for example, the continuous coordinate $\varphi$ shall be represented in discrete values $\varphi_{0}, \varphi_{1}, \varphi_{2}, \ldots, \varphi_{n-1}, \varphi_{n}$. Here, $\varphi_{0}$ is the initial value of a function $\varphi$, and $\varphi_{n}$ is its end (extreme) value. Similarly, functions $\dot{\varphi}, \ddot{\varphi}, \dddot{\varphi}, \stackrel{(4)}{\varphi}, \stackrel{(5)}{\varphi}$ shall be replaced with discrete values.

Then extreme conditions (15) are formulated as follows:

$$
\begin{cases}t=t_{0}=0: & \left\{\begin{array}{l}
\varphi=\varphi_{0}=0, \dot{\varphi}=\dot{\varphi}_{0}=0, \ddot{\varphi}=\ddot{\varphi}_{0}=0, \\
\dddot{\varphi}=\varphi_{0}=0, \stackrel{(4)}{\varphi}=\stackrel{(4)}{\varphi}_{0}=-\frac{M_{1} g}{I_{1} l}, \stackrel{(5)}{\varphi}=\stackrel{(5)}{\varphi}_{0}=0
\end{array}\right. \\
t=t_{n}: & \left\{\begin{array}{l}
\varphi=\varphi_{n}=\frac{\omega t_{n}}{2}, \dot{\varphi}=\dot{\varphi}_{n}=\omega, \ddot{\varphi}=\ddot{\varphi}_{n}=0, \\
\dddot{\varphi}=\dddot{\varphi}_{n}=0, \stackrel{(4)}{\varphi}=\stackrel{(4)}{\varphi}_{n}=-\frac{M_{1} g}{I_{1} l}, \stackrel{(5)}{\varphi}=\stackrel{(5)}{\varphi}_{n}=0 .
\end{array}\right.\end{cases}
$$

In the middle of the interval $\left[t_{0}, t_{n}\right]$ without extreme values, let us set the discrete values of a function $\varphi$ from $\varphi_{1}, \varphi_{2}$, up to $\varphi_{n-1}$, as shown in Fig. 2.

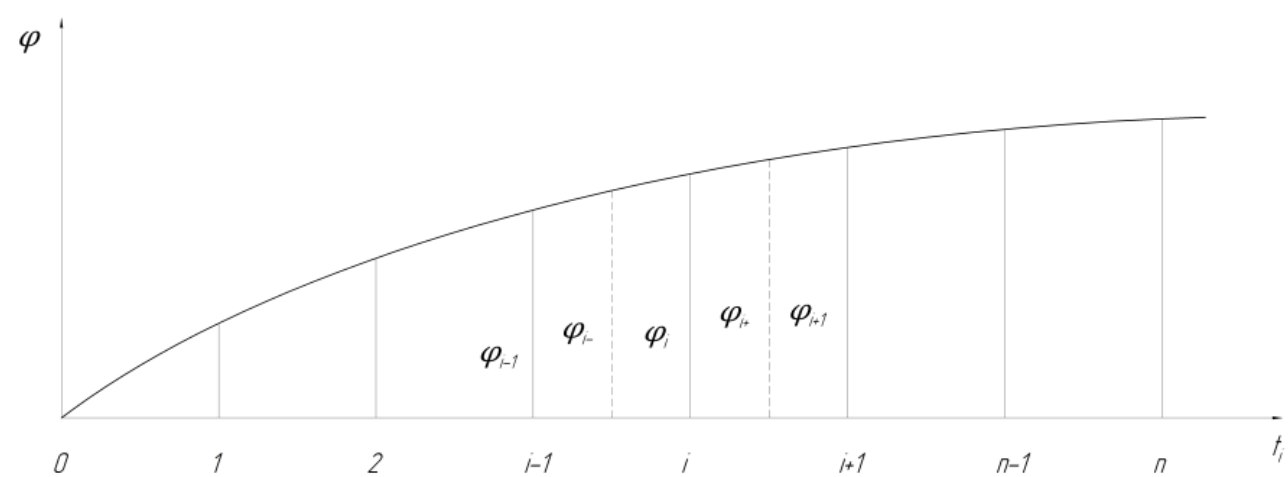

Fig. 2. Function representation with discrete values.

In the middle of the interval, the discrete values of the function are determined by dependences

$$
\varphi_{i-}=\left(\varphi_{i-1}+\varphi_{i}\right) / 2 ; \quad \varphi_{i+}=\left(\varphi_{i}+\varphi_{i+1}\right) / 2 .
$$


For discrete values of a function $\varphi_{i-1}, \varphi_{i-}, \varphi_{i}, \varphi_{i+}, \varphi_{i+1}$, when " $i$ " changes from 1 to $n-1$, discrete values of time derivatives of this function are calculated and used for the criterion:

$$
\begin{aligned}
& \dot{\varphi}_{i-}=\frac{\varphi_{i}-\varphi_{i-1}}{\Delta t} ; \quad \dot{\varphi}_{i+}=\frac{\varphi_{i+1}-\varphi_{i}}{\Delta t} ; \quad \dot{\varphi}_{i}=\frac{\dot{\varphi}_{i-}+\dot{\varphi}_{i+}}{2} ; \\
& \ddot{\varphi}_{i}=\frac{\dot{\varphi}_{i+}-\dot{\varphi}_{i-}}{\Delta t} ; \\
& \dddot{\varphi}_{i-}=\frac{\ddot{\varphi}_{i}-\ddot{\varphi}_{i-1}}{\Delta t} ; \quad \dddot{\varphi}_{i+}=\frac{\ddot{\varphi}_{i+1}-\ddot{\varphi}_{i}}{\Delta t} ; \quad \dddot{\varphi}_{i}=\frac{\varphi_{i-}+\varphi_{i+}}{2} ; \\
& \stackrel{(4)}{\varphi}_{i}=\frac{\dddot{\varphi}_{i+}-\dddot{\varphi}_{i-}}{\Delta t} \\
& \stackrel{(5)}{\varphi}_{i-}=\frac{\stackrel{(4)}{\varphi}_{i}-\stackrel{(4)}{\varphi}_{i-1}}{\Delta t} ; \quad \stackrel{(5)}{\varphi}_{i+}=\frac{\stackrel{(4)}{\varphi}_{i+1}-\stackrel{(4)}{\varphi}_{i}}{\Delta t} ; \quad \stackrel{(5)}{\varphi}_{i}=\frac{\stackrel{(5)}{\varphi}_{i-}+\stackrel{(5)}{\varphi}_{i+}}{2}
\end{aligned}
$$

By using the extreme conditions (16) and the discrete values of the function $\varphi_{1}$, $\varphi_{2}, \ldots, \varphi_{n-1}$, as well as dependencies $(17), \ldots,(22)$, the value of the optimization criterion may be determined

$$
\begin{aligned}
K=\frac{1}{M_{n} \sqrt{t_{n}}}\left\{\sum_{i=0}^{n-1}\{\right. & \delta\left[\left(I_{1}+m r^{2}\right) \ddot{\varphi}_{i}+I_{1} \frac{l}{g} \frac{(4)}{\varphi}\right]_{i}^{2} \\
& \left.\left.\left.+(1-\delta) \Delta t^{2}\left[\left(I_{1}+m r^{2}\right) \dddot{\varphi}_{i}+I_{1} \frac{l(5)}{g}\right)_{i}\right]^{2}\right\} \Delta t_{i}\right\}^{1 / 2} .
\end{aligned}
$$

By using the ME-PSO method, the determination of new values $\varphi_{1}, \varphi_{2}, \ldots, \varphi_{n-1}$ is carried out until the criterion (23) reaches its minimum value. The end discrete values of the function will signify the optimal mode of the boom system swing during the starting process.

The calculations have been made for the swing mechanism of a QTZ-80 boom crane with main parameters: inertia moment of the drive mechanism $I_{0}=7,1626.12$ kg. $\mathrm{m}^{2}$; inertia moment of the revolving part of the crane $I_{1}=4,920,738 \mathrm{~kg} \cdot \mathrm{m}^{2}$; the rigidity factor of the drive mechanism $C=6,626,669 \mathrm{Nm} / \mathrm{rad}$; mass of the load on a flexible suspension $m=2,000 \mathrm{~kg}$; length of the boom from the revolution axis of the crane to the point of load suspension on a flexible suspension $r=40 \mathrm{~m}$; length of the flexible load suspension $l=30 \mathrm{~m}$; free-fall acceleration $g=9.81 \mathrm{~m} / \mathrm{s}^{2}$; torque rating on the drive motor shaft reduced to the crane revolution axis $M_{n}=36.8 \mathrm{Nm}$; set angle rate of the crane swing $\omega=0.07 \mathrm{rad} / \mathrm{s}$; duration of a transient process (starting, braking) $t_{1}=6 \mathrm{~s}$; the resistance torque in the revolving part of the crane 


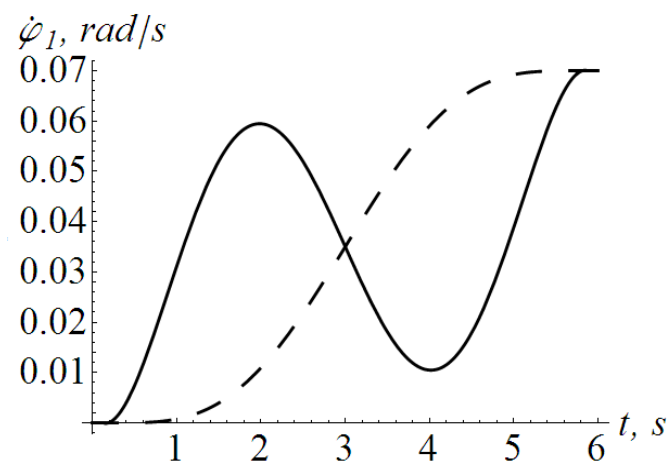

- of the revolving part

- - - - of the load

Fig. 3. Dependences of angle rate changes.

relative to its own revolution axis $M_{1}=0$. As a result of the solution, the optimal motion mode has been found, which is represented graphically (Fig. 3 - Fig. 5).

We can see from the graphical dependences of changes in the angle rate of the revolving part and the load (Fig. 3) obtained after solving the optimization problem using a complex dimensionless integral dynamic criterion, which takes into account the relative root-mean-square value of the elastic torque in the drive mechanism and its rate, that the load speed within the pre-set 6-second interval is accelerated to the rated value of $0.07 \mathrm{rad} / \mathrm{s}$ smoothly, without any oscillations in the crane-load boom system as contrasted to the "natural" (uncontrolled) characteristic, as shown in [2]. It allows achieving a considerable reduction in both the forces in the pillar (Fig. 4) during the transient processes and the oscillations of the load (Fig. 5).

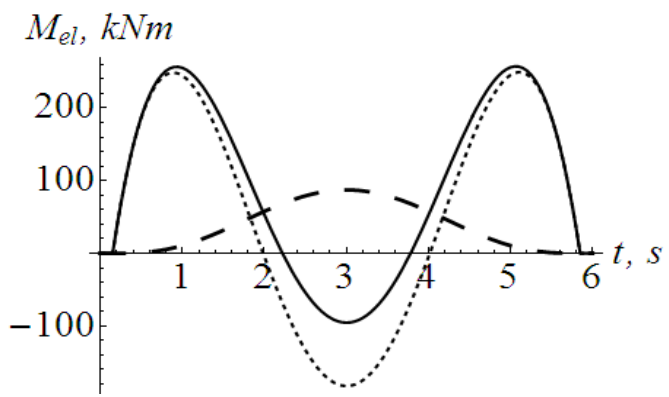

... The torque caused by inertia forces

- The torque in the pillar

- The torque caused by the pendulum oscillations of the weight on a flexible suspension

Fig. 4. Dependences of torques changes. 


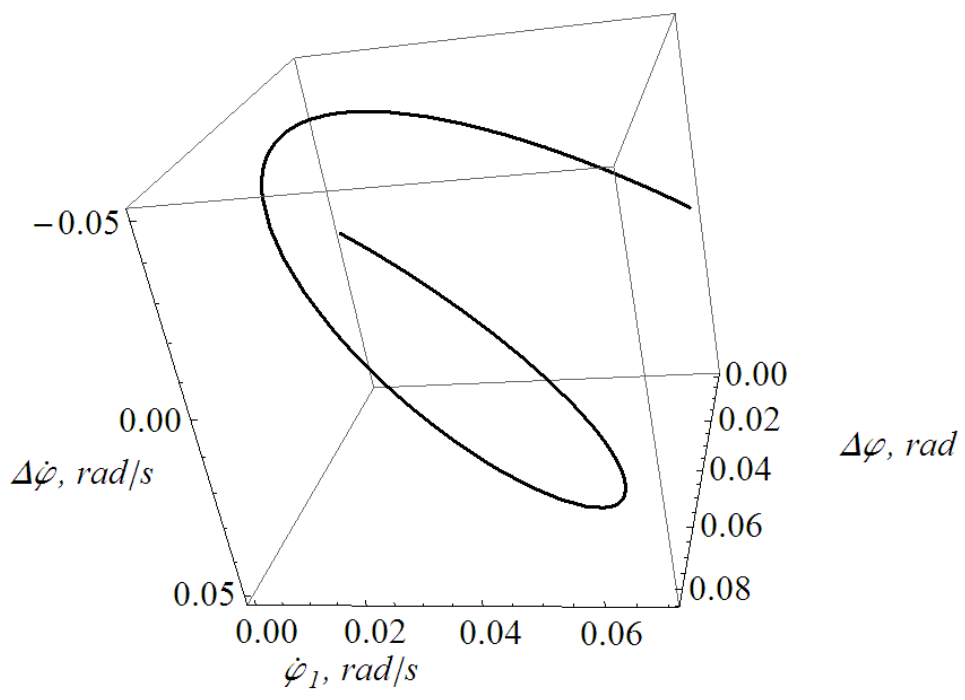

Fig. 5. 3D phase portrait.

\section{CONCLUSIONS}

1. In order to optimize the swinging mode of the load-lifting crane boom system, a three-mass dynamic model with known motion equations [2] has been chosen.

2. The complex integral dynamic criterion for the optimization of the crane swinging mode, which is represented in the form of a nonlinear integral functional, has been justified. The obtained criterion reflects the undesirable properties of the boom system, so its value has been minimized.

3. The nonlinear optimization problem of the boom crane swinging mode has been solved by minimizing the nonlinear integral functional. In order to solve the marginal problem of functional minimization, an approximate method has been used, namely the ME-PSO method modified by the authors. This method has made it possible to optimize the motion modes of nonlinear mechanical systems.

For the solution of the problem, the continuous functions $\varphi_{0}, \varphi_{1}, \varphi_{2}$ have been substituted by unknown discrete values. They were received as the input the ME-PSO algorithm and these values related to the global minimum criterion (23). Such result was provided by the strategy of searching of global extremum by the particle swarm and re-initialization of the swarm in case of its stagnation. 
4. The obtained optimal swinging mode of the crane boom system has made it possible to minimize the dynamical forces in the drive mechanism and the metal structures of the crane and eliminate any oscillations of the load on a flexible suspension during the transient process, which improves the performance in terms of reliability and efficiency in general.

5. It is recommended to implement the obtained optimal boom crane swinging mode using a mechatronic drive motor control system.

6. The above findings can be used to specify and improve the existing engineering methods for the calculation of the swing mechanisms of boom cranes with a flexible load suspension both during their design (engineering) and during every-day operation.

\section{REFERENCES}

[1] O. Grigorov, E. Druzhynin, G. Anishchenko, M. Strizhak, V. StrizhaK (2018) Analysis of Various Approaches to Modeling of Dynamics of Lifting-Transport Vehicles. International Journal of Engineering \& Technology 7(4) 64-70; DOI: 10.14419/ijet.v7i4.3.19553.

[2] V. Loveikn, S. Pylypaka, I. Kadykalo (2017) Dynamic Analysis of the Mechanism of Rotation of the Jib Crane. Scientific Herald of National University of Life and Enviromental Science of Ukraine. Series: Technique and energy of APK, Kiev 258 192-202.

[3] W. Shengchun, S. Rongsheng, J. Tonghong, S. Shijun (2012) Dynamic Behavior Analysis and Its Application in tower Crane Structure Damage Identification. Advanced Materials Research 368-373 2478-2482; DOI: https://doi.org/10.4028/www.scientific.net/AMR.368-373.2478.

[4] G. Rong, Y. Jing, L. Gang, Y. CongXun (2013) The Simulation of Rotary Motion of the Flexible Multi-Body Dynamics of Tower Crane. Advanced Materials Research 655-567 281-286; DOI: https://doi.org/10.4028/www.scientific.net/AMR.655-657.281.

[5] Y. Romacevych, V. Loveikin, O. Stekhno (2019) Closed-Loop Optimal Control of a System "Trolley - Payload" UPB Scientific Bulletin, Series D: Mechanical Engineering 81(2) 3-12.

[6] V. Loveikn, Yu. Romasevych (2017) Dynamic Optimization of a Mine Winder Acceleration Mode. Scientific Bulletin of National Mining University Scientific and Technical Journal 4 55-61.

[7] V. Loveikin, Yu. Loveikin, I. Kadykalo (2017) Analysis of Modes of Motion of Rotation Mechanism of Jib Crane. TEKA. An International Quarterly Journal on Motorization, Vehicle Operation, Energy Efficiency and Mechanical Engineering 18(1) $15-25$.

[8] L. Gulianitsky, O. Mulessa (2016) "Applied Methods of Combinatorial Optimization: Teach. Manual". Kyiv, Publishing and printing center "Kyiv University". 
[9] O. Bozorg-Haddad, M. Solgi, H. Loiciga (2017) "Meta-Heuristic and Evolutionary Algorithms for Engineering Optimization”. Hoboke, USA, John Wiley \& Sons Inc.

[10] J. Kennedy, R. Eberhart (1995) Particle Swarm Optimization. In: Proceedings of EEE International Conference on Neural Networks, pp. 1942-1948.

[11] F. Dubrovka, D. VAsilenko (2009) Constructive Synthesis of Planar Antennas Using Natural Optimization Algorithms. Izvestiya High Schools, Radio Electronics 4 3-22.

[12] S. Kiranyaz, T. Ince, A. Yildirim, M. Gabbouj (2009) Evolutionary Artificial Neural Networks by Multi-Dimensional Particle Swarm Optimization. Neural Networks 22(10) 1448-1462; DOI: 10.1016/j.neunet.2009.05.013.

[13] J. Heo, K. Lee, R. Garduno-Ramirez (2006) Multiobjective Control of Power Plants Using Particle Swarm Optimization Techniques. IEEE Transactions on Energy Conversion 21(10) 552-561; DOI: 10.1109/TEC.2005.858078.

[14] M. Zamani, M. Karimi-Ghartemani, N. Sadati, M. Parniani (2009) Design of a Fractional Order PID Controller for an AVR Using Particle Swarm Optimization. Control Engineering Practice 17(12) 1380-1387; DOI: 10.1016/j.conengprac.2009.07.005.

[15] A. Chander, A. Chatterjee, P. Siarry (2011) A New Social and Momentum Component Adaptive PSO Algorithm for Image Segmentation. Expert Systems with Applications 38(5) 4998-5004; DOI: 10.1016/j.eswa.2010.09.151.

[16] Y. Romasevych, V. Loveikin (2018) A Novel Multi-Epoch Particle Swarm Optimization Technique. Cybernetics and Information Technologies 18(3) 62-74; DOI: 10.2478/cait-2018-0039.

[17] V. Loveikin, Y. Romasevich, A. Khoroshun, A. Shevchuk (2018) TimeOptimal Control of a Simple Pendulum with a Movable Pivot, Part 1, International Applied Mechanics 54(3) 358-365; DOI: 10.1007/s10778-018-0887-x. 